\title{
Extracellular Matrix Elasticity Regulates Osteocyte Gap Junction Elongation: Involvement of Paxillin in Intracellular Signal Transduction
}

\author{
Demao Zhang Chenchen Zhou Qingxuan Wang Linyi Cai Wei Du \\ Xiaobing Li Xuedong Zhou Jing Xie
}

State Key Laboratory of Oral Diseases, West China Hospital of Stomatology, Sichuan University, Chengdu, China

\section{Key Words}

Substrate $\cdot$ Stiffness $•$ Osteocyte $\cdot$ Cytoskeleton $\cdot$ Connexin $43 \cdot$ Paxillin

\begin{abstract}
Background/Aims: Osteocytes can sense and respond to extracellular stimuli, including biochemical factors throughout the cell body, dendritic processes, and cilia bending. However, further exploration is required of osteocyte function in response to substrate stiffness, an important passive mechanical cue at the interface between osteocytes and the extracellular matrix, and the deep bio-mechanism in osteocytes involving mechanosensing of cell behavior. Methods: We fabricated silicon-based elastomer polydimethylsiloxane substrates with different stiffnesses but with the same surface topologies. We then seeded osteocytes onto the substrates to examine their responses. Methodologies used included scanning electron microscopy (SEM) for cell morphology, confocal laser scanning microscopy (CLSM) for protein distribution, western blot for protein levels, co-immunoprecipitation for protein interactions, and quantitative real-time polymerase chain reaction for gene expression. Results: SEM images revealed that substrate stiffness induced a change in osteocyte morphology, and CLSM of F-actin staining revealed that substrate stiffness can alter the cytoskeleton. These results were accompanied by changes in focal adhesion capacity in osteocytes, determined via characterization of vinculin expression and distribution. Furthermore, on the exterior of the cell membrane, fibronectin was altered by substrate stiffness. The fibronectin then induced a change in paxillin on the inner membrane of the cell via protein-protein interaction through transmembrane processing. Paxillin led to changes in connexin 43 via protein-protein binding, thereby influencing osteocyte gap junction elongation. Conclusion: This process from mechanosensing and mechanotransduction to cell function - not only indicates that the effects of mechanical factors on osteocytes can be directly sensed from the cell body, but also indicates the involvement of paxillin transduction.




\section{Cellular Physiology Cell Physiol Biochem 2018;51:1013-1026

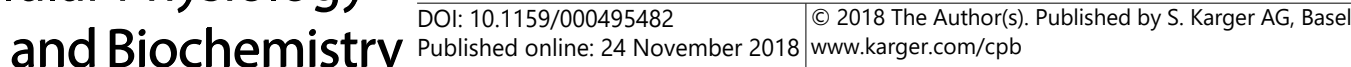 \\ Zhang et al.: Paxillin is Important in Stiffness-Triggered Gap Junction}

\section{Introduction}

Cells respond not only to forces that are actively applied to the extracellular matrix (ECM), but also to passive mechanical cues from the ECM itself, such as inherent stiffness $[1,2]$. Changes in passive mechanical factors, independent of the type or density of the chemical and molecular components in the ECM microenvironment, can affect cell shape, cytoskeletal reconstruction, cell phenotype maintenance, cell adhesion, cell migration, and stem cell differentiation [3-5]. For example, during embryogenesis, the mechanical cues can mold the ECM structure and alter the level of cytoskeletal tension within the growing cells, thereby changing the cell growth patterns and influencing tissue development [6, 7]. During the postnatal development of tissues, the mechanical cues thicken stress fibers, which are prominent during periods of cellular elongation, and permit an oriented deposition of ECM proteins that establish a scaffold for the architecture of the mature tissue [8]. During tumourigenesis, ECM stiffness directs integrin clustering, focal adhesion change, stress fiber formation, and ultimately malignant transformation [9]; thus, ECM stiffness provides a subcellular explanation for the clinical correlation between high gross tumor stiffness and poor prognosis. Although knowledge concerning the interface between cells and the ECM is rapidly advancing, further investigations of the precise regulatory mechanisms that enable cells to respond to matrix biophysical properties are necessary.

Osteocytes, one of the most important residents in bone tissue, are capable of sensing mechanical cues applied to the bone; they then react to these loads by controlling the inner metabolic signals of the bone and the activities of osteoblasts (bone-forming cells) and osteoclasts (bone-resorbing cells) through cell-to-cell communication or secreted factors [10-12]. Mechanical stimulation of osteocytes alters their metabolic activity. For example, osteocytes start to produce signaling molecules, such as Wnts, prostaglandin E2, bone morphogenetic proteins, and nitric oxide, enabling a rebalance of the bone metabolism and the recruitment of osteoblasts and osteoclasts $[13,14]$. Furthermore, osteocytes are capable of quickly changing their enzyme activity and RNA synthesis in the intact bone after mechanical loading $[15,16]$. Ablation of $80 \%$ of the osteocytes prevents the bone loss typically seen after unloading [17]. Thus, an investigation of the effect of mechanical stimuli on osteocyte behavior is essential, as well as an exploration of the fundamental mechanism of active mechanosensing and mechanotransducing by osteocytes in cell-matrix interactions.

In this study, we fabricated substrates with different stiffnesses but with the same surface topology based on silicon-based elastomer polydimethylsiloxane (PDMS) and explored osteocyte behavioral changes such as cell morphology, cytoskeleton, focal adhesion ability, ECM protein anchoring, protein mechanotransduction, and the gap junction in response to substrate stiffness. Determination of the cell behavioral changes from active mechanosensing and mechanotransduction to the osteocyte gap junction in response to substrate stiffness will help to increase understanding of the mechanism underlying the interface between cells and their ECM microenvironment.

\section{Materials and Methods}

\section{Preparation of PDMS substrates}

PDMS has been used as a principal candidate in many biomaterial studies because it has advantages such as flexibility, optical clarity, and elastic tenability $[18,19]$. In this study, PDMS substrates were prepared by mixing Sylgard 184 (Corning, Corning, NY), as curing agent, in five different ratios to the oligomeric base (i.e., 1:5, 1:15, 1:30, 1:45, and 1:60). The mixtures were subsequently cast onto a group of single-well Petri dishes (35 × $10 \mathrm{~mm}$; Corning) and cured at $60^{\circ} \mathrm{C}$ for $3 \mathrm{~h}$. This was followed by a sterilization step, which entailed the exposure of samples to UV radiation for $2 \mathrm{~h}$, and cell culture. 


\section{Cellular Physiology Cell Physiol Biochem 2018;51:1013-1026 and Biochemistry \begin{tabular}{l|l} 
DOI: $10.1159 / 000495482$ & 2018 The Author(s). Published by S. Karger AG, Basel
\end{tabular}

\section{Young's modulus measurements of PDMS substrates}

Young's modulus measurement was performed using the spherical indentation method to characterize the stiffnesses of different PDMS substrates, as described previously [19]. Briefly, the ElectroForce $₫ 3100$ test instrument (Bose, Shanghai, China) was used for the indentation tests. A spherical indenter with a radius of $3 \mathrm{~mm}$ was used, and the measurements were performed in ambient conditions. Loading procedures were undertaken with displacement controlled; the loading rate was set at $2 \mathrm{~mm} / \mathrm{s}$ while the maximum indentation depth was $3 \mathrm{~mm}$. Samples had a diameter of $55 \mathrm{~mm}$ and height of $15 \mathrm{~mm}$. Six measurements at different positions of the sample were obtained, and depth-indentation load curves were recorded. The initial shear modulus was determined by fitting the load curves up to different ratios of $\mathrm{h} / \mathrm{R}$ using the Hertzian and Hyperelastic solutions [19], as follows:

$$
P=\frac{16}{9} E \sqrt{R h h}\left(1-0.15 \frac{h}{R}\right)
$$

where $E$ represents the Young's modulus, $P$ the indentation load, $h$ the indentation depth, and $R$ the indenter radius.

\section{Surface topographic characterization of PDMS substrates}

The surfaces of PDMS membranes with different stiffnesses were imaged by atomic force microscopy (AFM) (Nanoscope IIIa; Digital Instruments, Santa Barbara, CA) in tapping mode with $512 \times 512$-pixel data acquisition. The scan speed was $1 \mathrm{~Hz}$ at ambient conditions. The topographic images were obtained with a standard silicon tip on a cantilever beam. The spring constant of the cantilever was $50 \mathrm{pN} / \mathrm{nm}$, and its length was $125 \mu \mathrm{m}$, with a resonant frequency of $300 \mathrm{kHz}$.

\section{Scanning electron microscopy}

For observation of morphological changes, osteocytes were seeded onto PDMS substrates with different stiffnesses at a lower density ( $30 \%$ confluence) for 3 days. The cells were then fixed with $2.5 \%$ glutaraldehyde for $2 \mathrm{~h}$ and dehydrated in a graded ethanol concentration of $50 \%, 60 \%, 70 \%, 80 \%, 90 \%$, $95 \%$, and $100 \%$. After dehydration, the samples were mounted on specimen holders, coated with a thin layer of gold, and then scanned by scanning electron microscopy (SEM).

\section{Cell culture}

The osteocyte cell line MLO-Y4 was purchased from the University of Texas (Austin, TX). The basal medium for the cell line is formulated Dulbecco's modified Eagle's medium. To make a complete growth medium, additional agents were added, including $4.5 \mathrm{~g} / \mathrm{L}$ glucose. The cells were then normally cultured with $10 \%$ fetal bovine serum medium with $0.1 \mathrm{mM}$ non-essential amino acids, $4 \mathrm{mM} \mathrm{L}$-glutamine, and $1 \%$ antibiotics and incubated at $37{ }^{\circ} \mathrm{C}$ in a humidified atmosphere of $5 \% \mathrm{CO}_{2}$ and $95 \%$ air.

\section{Confocal laser scanning microscopy}

To visualize cytoskeletal and protein changes in response to different substrate stiffnesses, osteocytes were seeded on PDMS substrates with different stiffnesses and equilibrated for $72 \mathrm{~h}$. Then, the cells in each group were washed three times using $1 \times$ phosphate-buffered saline (PBS) and fixed in $4 \%$ (w/v) paraformaldehyde for $1 \mathrm{~h}$. The fixed cells were washed with $1 \times$ PBS until the smell of paraformaldehyde vanished. Bovine serum albumin $(5 \%(\mathrm{w} / \mathrm{v}))$ was applied for $1 \mathrm{~h}$ as a block and the samples were then stained with FITC-phalloidin (6.6 $\mu \mathrm{M}$; Invitrogen, Carlsbad, CA) or protein antibodies for 1-2 $\mathrm{h}$ at room temperature (RT) (or incubated overnight at $4{ }^{\circ} \mathrm{C}$; the used FITC-phalloidin and antibodies were recycled according to the manufacturer's instructions). The next step was the removal of the FITC-phalloidin and antibodies and sealing with block buffer (Invitrogen). The cytoskeletons of cell samples were observed using a confocal laser scanning microscope (A1R MP+; Nikon, Tokyo, Japan; parameters: $20 \times$, Nikon Microsystems original image: $1024 \times 1024,100 \mu \mathrm{m}$ ). Cell spreading areas were calculated using Image-Pro Plus 6.0 (Media Cybernetics, Rockville, MD). The changes in numbers, lengths, and widths of gap junctions among osteocytes in response to various substrate stiffnesses were calculated based on at least five osteocytes per image by confocal laser scanning microscopy (CLSM) at 20× magnification in each experiment. The experiments were performed at least three times. 


\section{Cellular Physiology Cell Physiol Biochem 2018;51:1013-1026

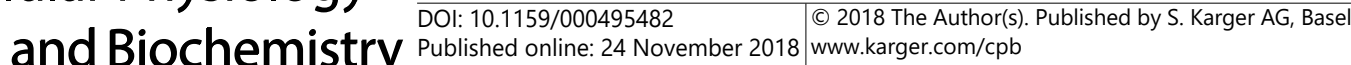 \\ Zhang et al.: Paxillin is Important in Stiffness-Triggered Gap Junction}

Western blot

Protein samples were prepared by mixing one part tissue sample with one part Bio-Rad Laemmli sample buffer (Bio-Rad Laboratories, Hercules, CA) and then boiled at $100{ }^{\circ} \mathrm{C}$ for 5 min. Proteins were separated by $8-12 \%$ sodium dodecyl sulfate-polyacrylamide gel electrophoresis (according to molecular weight) and transferred to a polyvinylidene fluoride (PVDF) membrane at $200 \mathrm{~mA}$ for $1 \mathrm{~h}$ at RT. The blot was blocked with 5\% non-fat dry milk suspended in $1 \times$ Tris-buffered saline with Tween 20 for $2 \mathrm{~h}$ at RT. The resulting blot was incubated with 1:500-5000 antibody dilutions according to the specific manufacturer's recommendations for $2 \mathrm{~h}$ at RT or overnight at $4{ }^{\circ} \mathrm{C}$, followed by incubation with 1:2000-10000 rabbit antigoat IgG-HRP from Santa Cruz Biotechnology (Santa Cruz, CA) for $2 \mathrm{~h}$ at RT. Signals from blots were obtained using the Western Blotting Luminol Reagent Kit (Santa Cruz Biotechnology). Proteins were visualized by a densitometer (Bio-Rad Laboratories) and a chemiluminescence kit (Lot No. 1723301, Millipore, Darmstadt, Germany).

\section{Co-immunoprecipitation}

Protein immunoprecipitation was performed using the Pierce ${ }^{\circledR}$ Co-immunoprecipitation Kit (Lot\#: SB240573B, Thermo Fisher Scientific, Waltham, MA). Briefly, osteocytes were lysed according to the manufacturer's instructions, and the antibody of the bait protein was added to the lysate at a 1:30 ratio. After overnight incubation, the antigen (bait protein) and the interacting proteins (prey proteins) were separated and purified with a centrifugal column provided in the kit. After collection with elution buffer, the samples were blotted onto the PVDF membrane via western blot.

\section{Quantitative real-time polymerase chain reaction}

Quantitative real-time polymerase chain reaction (qPCR) was performed as previously described [20]. To evaluate the expression levels of connexin 43, qPCR was performed with the SYBR Green PCR Kit using iCycler (Bio-Rad Laboratories). PCR reactions were performed at $0.5 \mathrm{mM}$ for each primer in a $25-\mu \mathrm{l}$ volume containing $1 \mu \mathrm{l}$ of the cDNA sample. The reaction was initiated by activating the polymerase with a 5 -min preincubation at $95^{\circ} \mathrm{C}$. Amplification was achieved with 45 cycles of $15 \mathrm{~s}$ of denaturation at $94{ }^{\circ} \mathrm{C}, 15 \mathrm{~s}$ of annealing at $65^{\circ} \mathrm{C}$, and $10 \mathrm{~s}$ of extension at $72^{\circ} \mathrm{C}$. The program was concluded by a melting curve analysis. All experiments were performed in triplicate. The copy numbers of each gene were determined with cycle threshold $(\Delta \Delta \mathrm{CT})$ methods. The means of the copy numbers of glyceraldehyde 3-phosphate dehydrogenase were used as internal controls. Standard curves of all primers were prepared from total normal cDNA, amplified by semiquantitative PCR, and cloned by use of the TOPO II TA Cloning Kit (K450002, Thermo Fisher Scientific) following the manufacturer's recommendations.

\section{Statistical analysis}

Statistical analysis was performed by one-way analysis of variance to determine whether differences existed among groups. Post hoc analyses were conducted using Fisher's protected least significant differences. In each analysis, the critical significance level was set at $\mathrm{p}<0.05$.

\section{Results}

Substrate stiffness alters the cell morphology of osteocytes

We designed the substrates using the silicon-based elastomer PDMS due to its advantages in biomaterial studies. By mixing the curing agent with the oligomeric base at 1:5, 1:15, $1: 30,1: 45$, and 1:60 ratios as previously described [18], a series of PDMS substrates was fabricated. Their surface topologies were first characterized by AFM (Fig. 1A). These PDMS substrates had smoother surfaces than those of the Petri dish control group (comprising unaltered Petri dishes). Root mean square analysis further confirmed the lower surface roughness in the PDMS substrates relative to the Petri dish control group (Fig. 1B). However, the PDMS substrates showed significantly different stiffnesses and their corresponding Young's elastic moduli are shown in Fig. 1C. The spreading areas of osteocytes attached in a monolayer culture were far broader than 1 micron $(>1 \mu \mathrm{m})$; herein, we ignored the impact of surface roughness and only considered the stiffness of the PDMS substrates as the 


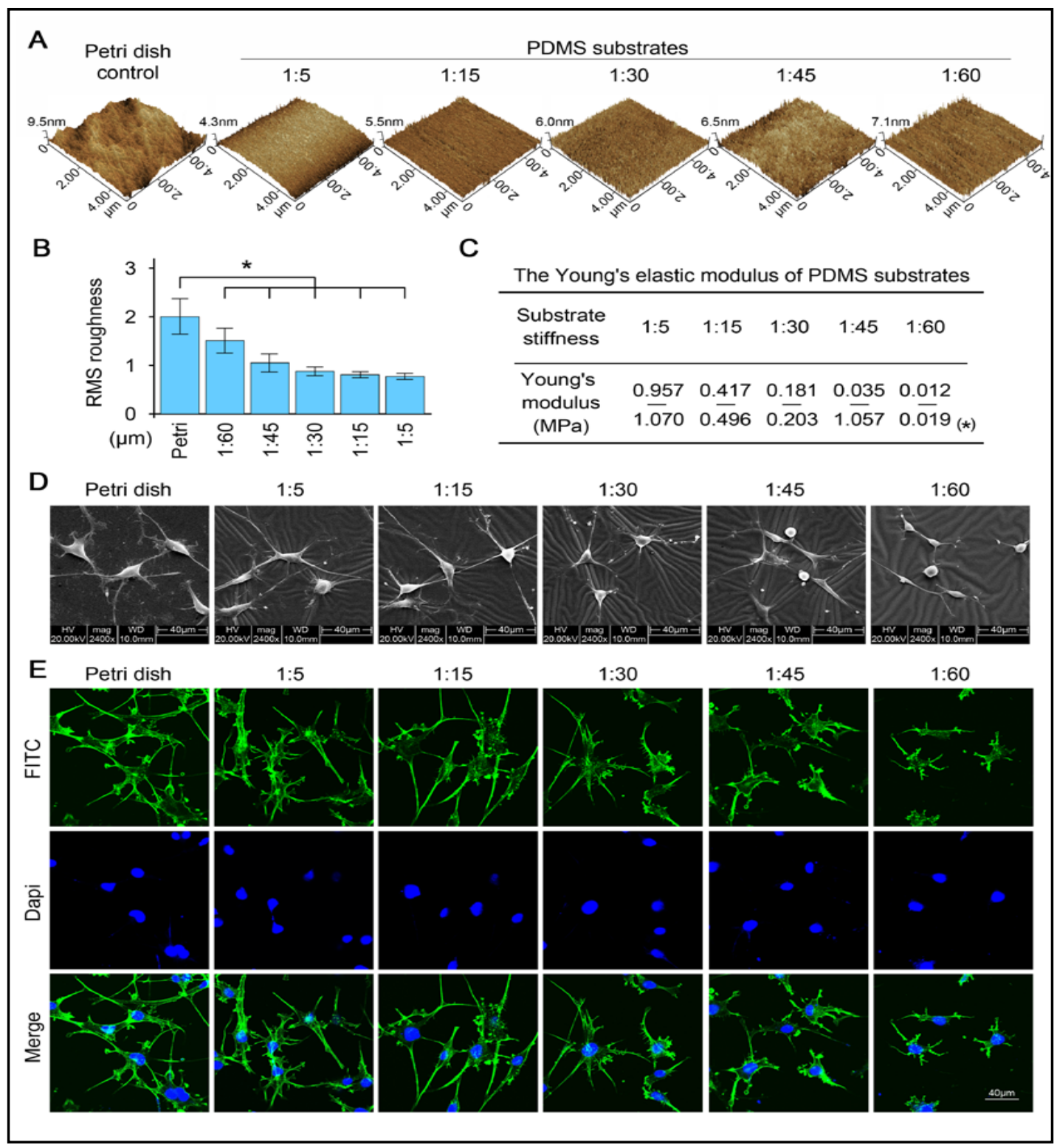

Fig. 1. Cell morphology changes in response to different PDMS substrate stiffnesses. (A) Representative AFM images of PDMS substrates. Each topographic image of the PDMS film was repeated at least six times $(n=6)$. (B) Characterization of the root mean square roughness of PDMS surfaces $(n=3)$ * Significant difference with respect to the Petri dish control $(\mathrm{p}<0.05)$. (C) Young's moduli of PDMS substrates. The data are the mean of six independent experiments $(n=6),{ }^{*} p<0.05$. (D) Representative morphology changes via SEM in osteocytes grown on PDMS substrates with different stiffnesses. (E) Representative cytoskeleton changes of osteocytes by F-actin staining in response to different substrate stiffnesses.

primary mechanical stimulus. To confirm the influence of substrate stiffness on osteocytes, we first seeded the osteocytes on these substrates and detected the basal cell morphology changes in osteocytes by SEM (Fig. 1D). The areas of cell spreading wee greatly reduced with a decrease in the substrate stiffness. These results were further confirmed through cytoskeleton staining via characterization of the F-actin distribution (Fig. 1E). 


\section{Substrate stiffness modulates vinculin expression}

We next explored the adhesion capacity of osteocytes based on the results of cell morphology changes in response to different substrate stiffnesses. We found that the distribution of vinculin, a membrane-cytoskeletal protein in focal adhesion plaques in charge of linking integrin adhesion molecules to the actin cytoskeleton, decreased as the substrate stiffness decreased (Fig. 2A). The western blot results confirmed the changes in vinculin protein in osteocytes in response to different substrate stiffnesses (Fig. 2B). The dispersion graph plotted using optical density (OD) analysis based on three independent experiments further showed the protein changes in vinculin (Fig. 2C). Quantification revealed the fold changes in vinculin expression (Fig. 2D): there was up to a $75 \%$ reduction in vinculin in the softest substrate group compared with the Petri dish group.

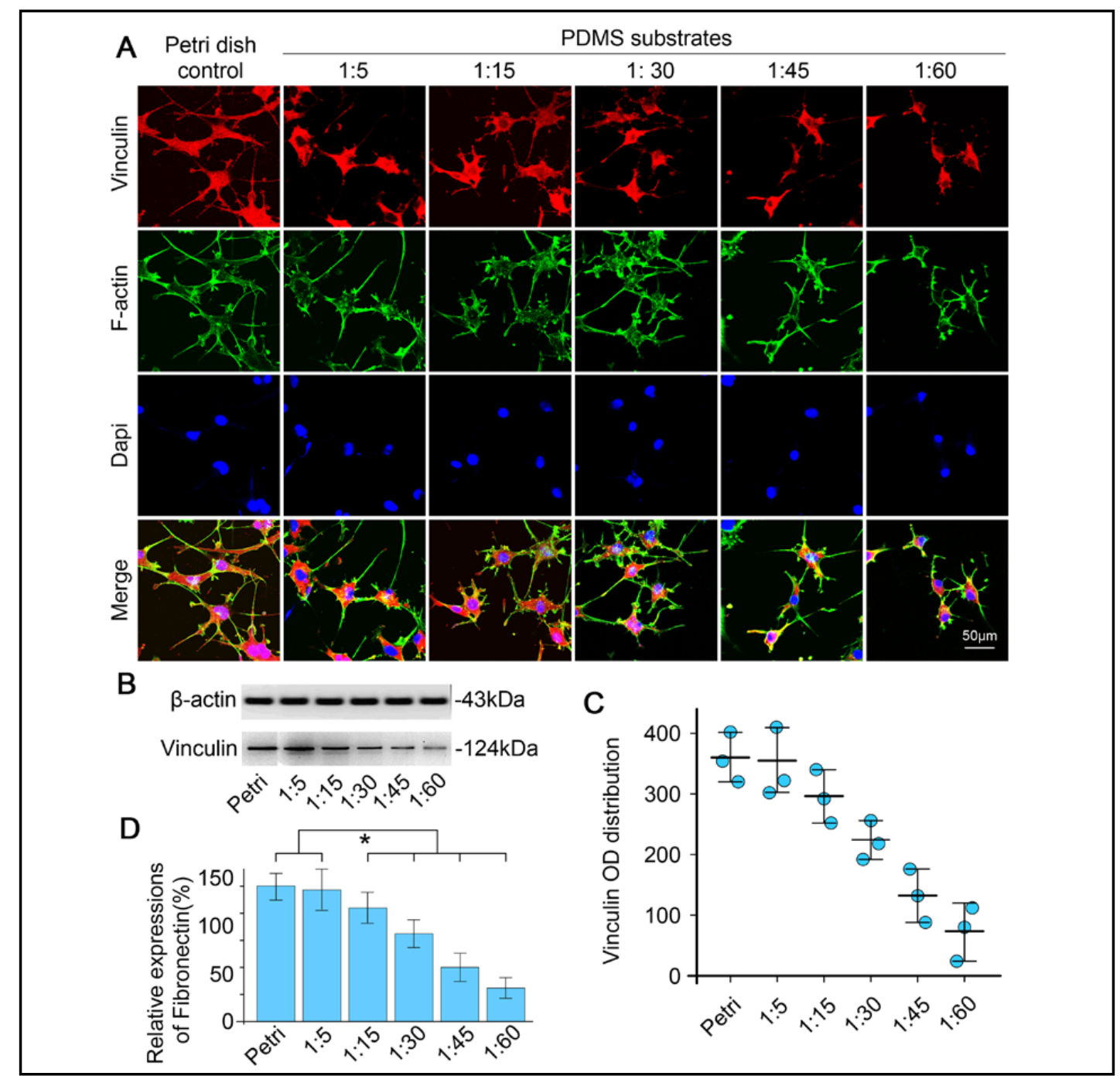

Fig. 2. Changes in vinculin in osteocytes in response to different PDMS substrate stiffnesses. (A) Representative images of vinculin by CLSM were taken 3 days after cell seeding. The results shown are representative of three repeated experiments $(n=3)$. Co-staining with F-actin was used as the cell shape control. (B) Protein expressions of vinculin by western blot in osteocytes grown on PDMS substrates with different stiffnesses. (C) Dispersion graph showing the changes in OD in western blot bands based on three independent repeats by Image-Pro Plus. (D) Quantitative analysis of vinculin expression by the western blot ratio to that of the Petri dish control group $(n=3)$. ${ }^{*}$ Significant difference with respect to the Petri dish control $(\mathrm{p}<0.05)$. 
Substrate stiffness regulates fibronectin and triggers intracellular signal transduction through paxillin

Fibronectin binds to membrane-spanning receptor proteins and integrins and functions as a link between the ECM and cell membrane [21]. We found coincident changes with the reduction in substrate stiffness through immunofluorescent staining analysis on CLSM (Fig. 3A). The distribution of fibronectin in osteocytes decreased in accordance with the reduction in cell spreading areas according to the F-actin stain. We next used western blot and found a reduction in total fibronectin in response to the reduced substrate stiffness (Fig. 3B). The dispersion graph from OD analysis further showed the changes in fibronectin protein (Fig. 3C). This reduction was further qualified in Fig. 3D. We also found that fibronectin was related to paxillin, a signal transduction adaptor protein with rich protein-protein interaction sites in the N-terminal region [22]. The interaction between fibronectin and paxillin was

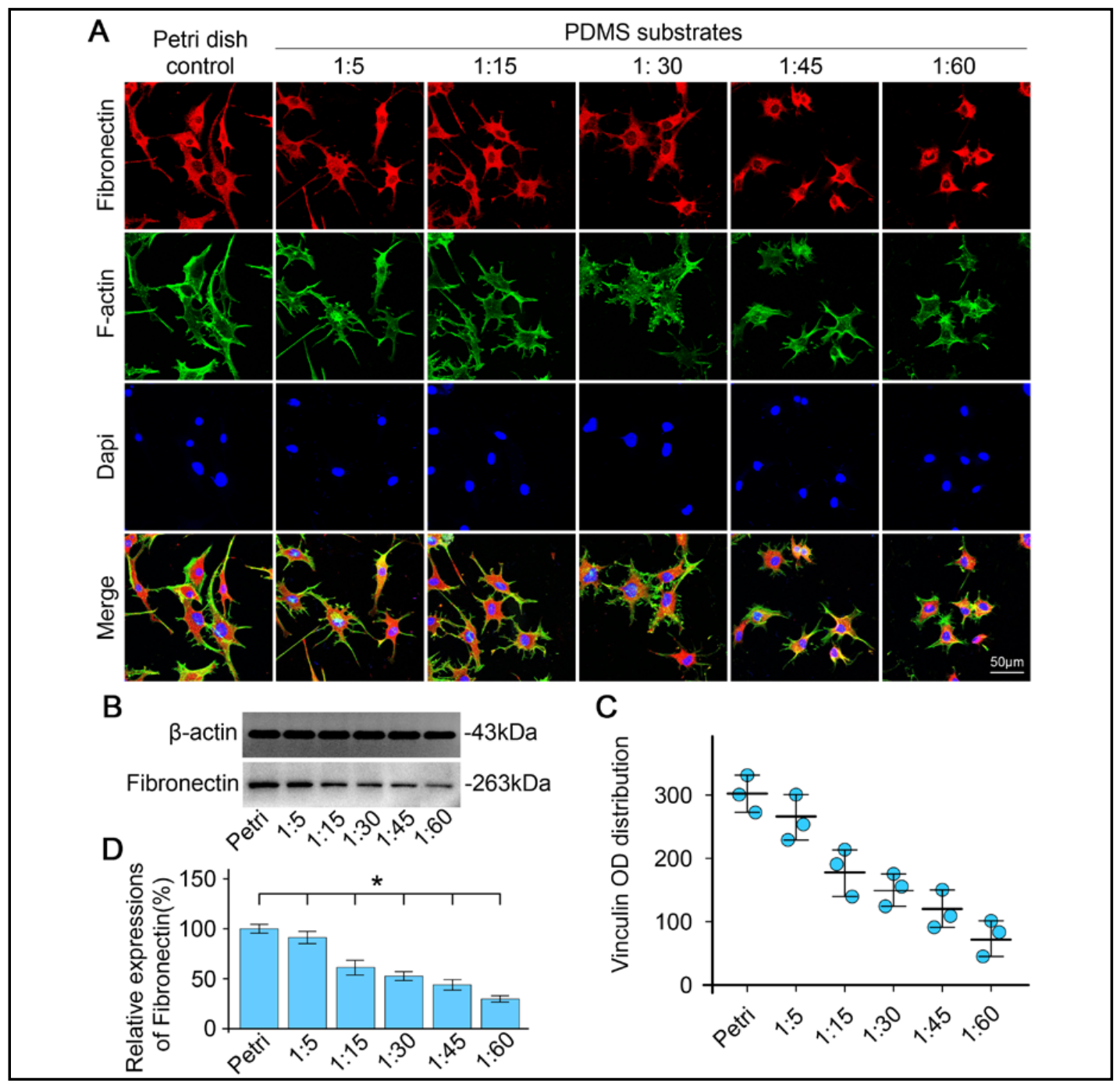

Fig. 3. Expression changes in fibronectin in response to different PDMS substrate stiffnesses. (A) Representative changes in fibronectin distribution by CLSM in osteocytes grown on PDMS substrates with different stiffnesses. (B) Protein expressions of fibronectin by western blot in osteocytes grown on PDMS substrates with different stiffnesses. (C) Dispersion graph showing the changes in OD in western blot bands based on three independent repeats by Image-Pro Plus. (D) Quantitative analysis of fibronectin expression by the western blot ratio to that of the Petri dish control group $(n=3) . *$ Significant difference with respect to the Petri dish control $(\mathrm{p}<0.05)$. 
confirmed via the co-immunoprecipitation (Co-IP) assay (Fig. 4A). An examination of the expressions of paxillin in response to substrate stiffness revealed that the total amounts of paxillin decreased as substrate stiffness decreased (Fig. 4B). The dispersion graph based on the three independent experiments further showed the protein changes in paxillin (Fig. 4C). Quantification confirmed the western blot results (Fig. 4D). We next explored the distribution of paxillin in osteocytes in response to substrate stiffness. CLSM images of the F-action-stained cytoskeleton revealed that paxillin is distributed along the cytoskeleton and that its level mirrors that of the cytoskeleton (Fig. 4E).

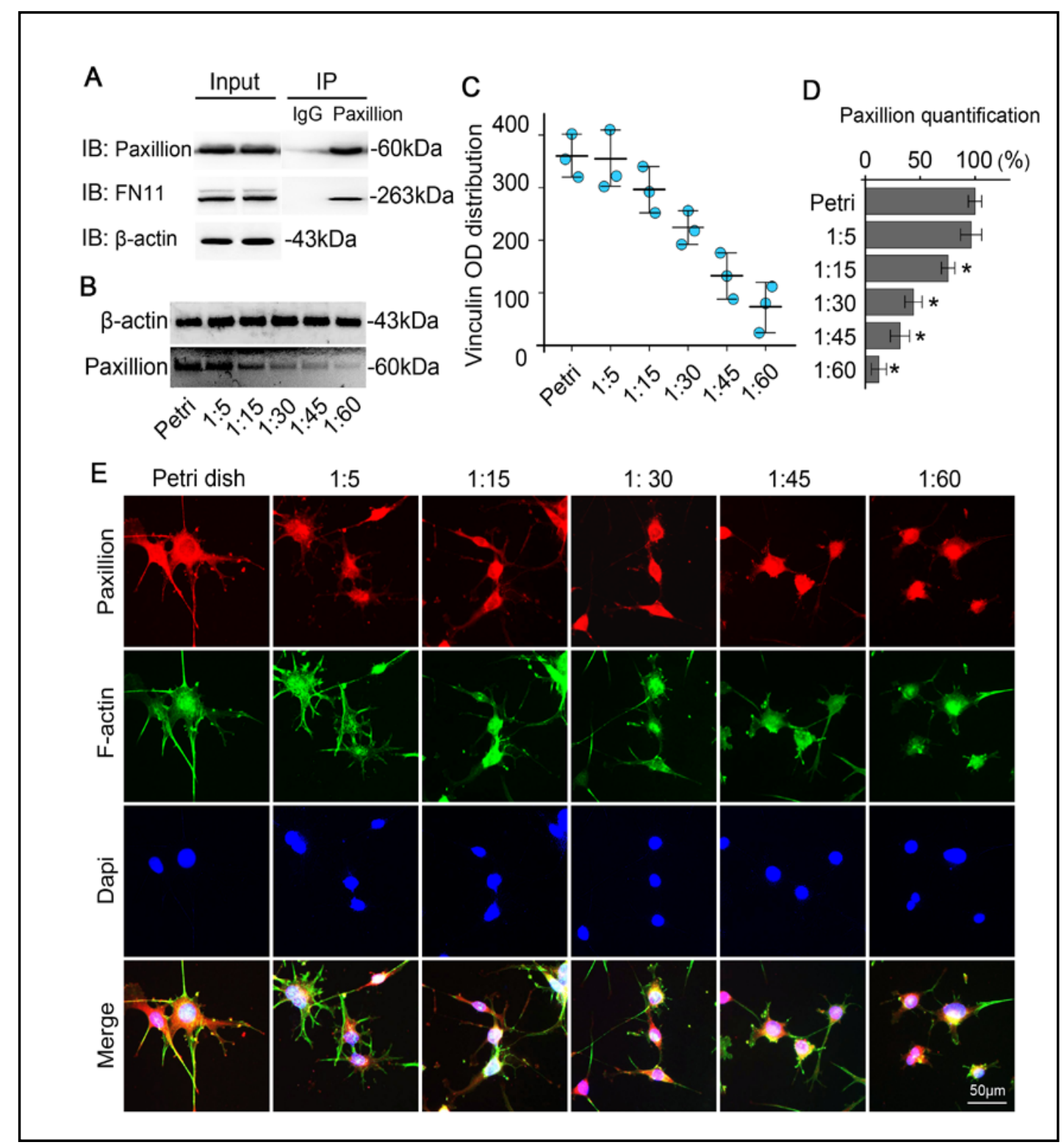

Fig. 4. Fibronectin triggers changes in paxillin located on the inner side of the intracellular membrane. (A) Co-IP showing the interaction of fibronectin and paxillin. $\beta$-actin was used as the internal control. (B) Decreased expressions of paxillin in response to reduced substrate stiffnesses on western blot. (C) Dispersion graph showing the changes in OD in western blot bands based on three independent repeats by Image-Pro Plus. (D) Quantitative analysis of paxillin expression by the western blot ratio to that of the Petri dish control group $(n=3)$. *Significant difference with respect to the Petri dish control $(\mathrm{p}<0.05)$. (E) Representative distribution of paxillin in osteocytes on different PDMS substrates by CLSM. 


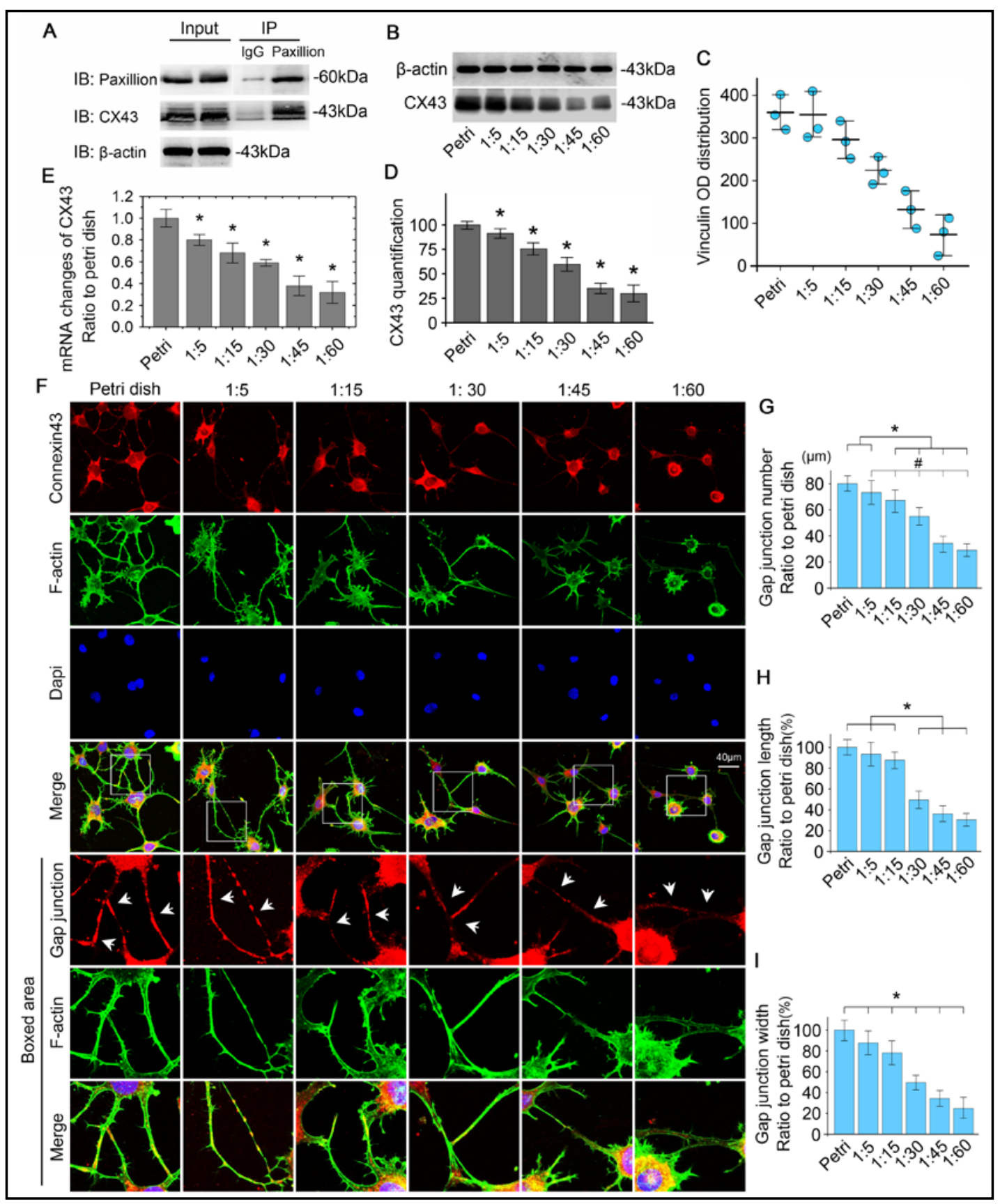

Fig. 5. Paxillin directly induces changes in connexin 43 in osteocytes in response to different PDMS substrate stiffnesses. (A) Co-IP showing the interaction of paxillin and connexin 43 . $\beta$-actin was used as the internal control. (B) Decreased expressions of connexin 43 in response to the reduced substrate stiffnesses on western blot. (C) Dispersion graph showing the changes in OD in western blot bands based on three independent repeats by Image-Pro Plus. (D) Quantitative analysis of connexin 43 expression by western blot ratio to that of the Petri dish control group $(n=3)$. *Significant difference with respect to the Petri dish control group ( $\mathrm{p}<0.05$ ). (E) qPCR showing the mRNA changes in connexin 43 in osteocytes in response to varied substrate stiffnesses. (F) Representative distribution of connexin 43 in osteocytes on different PDMS substrates by CLSM. The boxed areas show the changes in gap junction between osteocytes by characterization of connexin 43. (G) Changes in gap junction number between osteocytes $(n=50)$. $(H)$ Changes in gap junction length between osteocytes $(n=50)$. (I) Changes in gap junction width between osteocytes $(\mathrm{n}=50)$. E-G, *Significant difference with respect to the Petri dish control group $(\mathrm{p}<0.05)$.

\section{KARGER}


Substrate stiffness modulates the gap junction elongation of osteocytes by concentrating connexin 43 through paxillin binding

Because osteocytes communicate with each other through the gap junction [23], we next explored the influence of substrate stiffness on the gap junction by examining connexin 43, whose distribution is reported in vitro [24] and in vivo [25]. At the protein level, we first found an interaction between paxillin and connexin 43 by Co-IP (Fig. 5A). The connexin 43 in osteocytes was reduced in response to a reduced substrate stiffness on western blot (Fig. 5B). Quantification confirmed the results of western blot seen in the dispersion graph (Fig. 5C) and histogram (Fig. 5D). At the mRNA level, we also found that the mRNA of connexin 43 is reduced in osteocytes in accordance with the decrease in substrate stiffness (Fig. 5E). To determine the location of connexin 43 in osteocytes, we further explored the distribution of connexin 43 and found that connexin 43 was expressed in the cytoplasm, especially along the cytoskeleton (F-actin), but was reduced along with the reduction in substrate stiffness (Fig. 5F). Additionally, from the boxed area in Fig. 5F, we can see the gap junction change between the osteocytes in response to substrate stiffness.

Finally, we performed quantitative analysis of the changes in the numbers, lengths, and widths of the gap junctions among osteocytes in response to varied substrate stiffnesses. By using Image-Pro Plus, the numbers, lengths, and widths of gap junctions among five osteocytes per image taken by CLSM $(20 \times)$ were calculated. The quantitative statistical analyses were based on approximately 50 osteocytes in three independent experiments. The numbers of gap junction were reduced with a decrease in substrate stiffness (Fig. 5G). The length (Fig. 5H) and width (Fig. 5I) both decreased as substrate stiffness decreased.

\section{Discussion}

Interactions between cells and the ECM regulate numerous cellular behaviors, including gene expression, protein synthesis, cell cycling, proliferation, and differentiation. On the one hand, cells are able to synthesize, breakdown, or otherwise rearrange ECM components to change ECM composition and topography. On the other hand, the biophysical properties of the ECM also play a role in directing cell functions and, thus, any changes in ECM dynamics will influence adjacent cells and their cellular activities [26,27]. By maintaining the feedback loop between cells and their ECM, tissues/cells can readily adapt to the physical forces imposed on them $[26,28]$. However, when the tissue homeostasis becomes imbalanced, tissue function generally becomes impaired as a result of aberrant cellular behavior [29]. Thus, it is critical to understand how cells respond to their mechanical environment for the treatment and prevention of diseases. In this study (Fig. 6, schematic diagram), based on PDMS substrates with different stiffnesses, we detected changes in cell morphology, the osteocyte cytoskeleton, and focal adhesion capacity by characterizing vinculin expression. We next found that fibronectin, a protein that connects the ECM and cell membrane, interacted with paxillin, a signal transduction adaptor protein that is capable of triggering intracellular signaling. We further found that paxillin interacted with connexin 43 , the marker protein for gap junction and that this interaction influenced the communication between osteocytes. These results can help to explain the modulatory mechanisms from primary mechanosensing to final cell function.

The osteocyte has several mechanisms through which it responds to the mechanical changes occurring in its environment, such as throughout the cell body, the dendritic processes, or the bending of cilia. Recently, it has been reported that the mechanosensing mechanism is initiated in the cell body [30], whereas mechanotransduction is initiated by another proteoglycan (i.e., glycocalyx), which is found on the surfaces of dendritic processes but not on the cell body [31]. Other research has suggested that the osteocyte senses mechanical stimuli through cilia, which are single flagellar-like structures found on every cell [32]. Furthermore, cilia in osteocytes do not mediate calcium flux in response to shear force of fluid flow but do induce the release of prostaglandin, which is different from kidney 


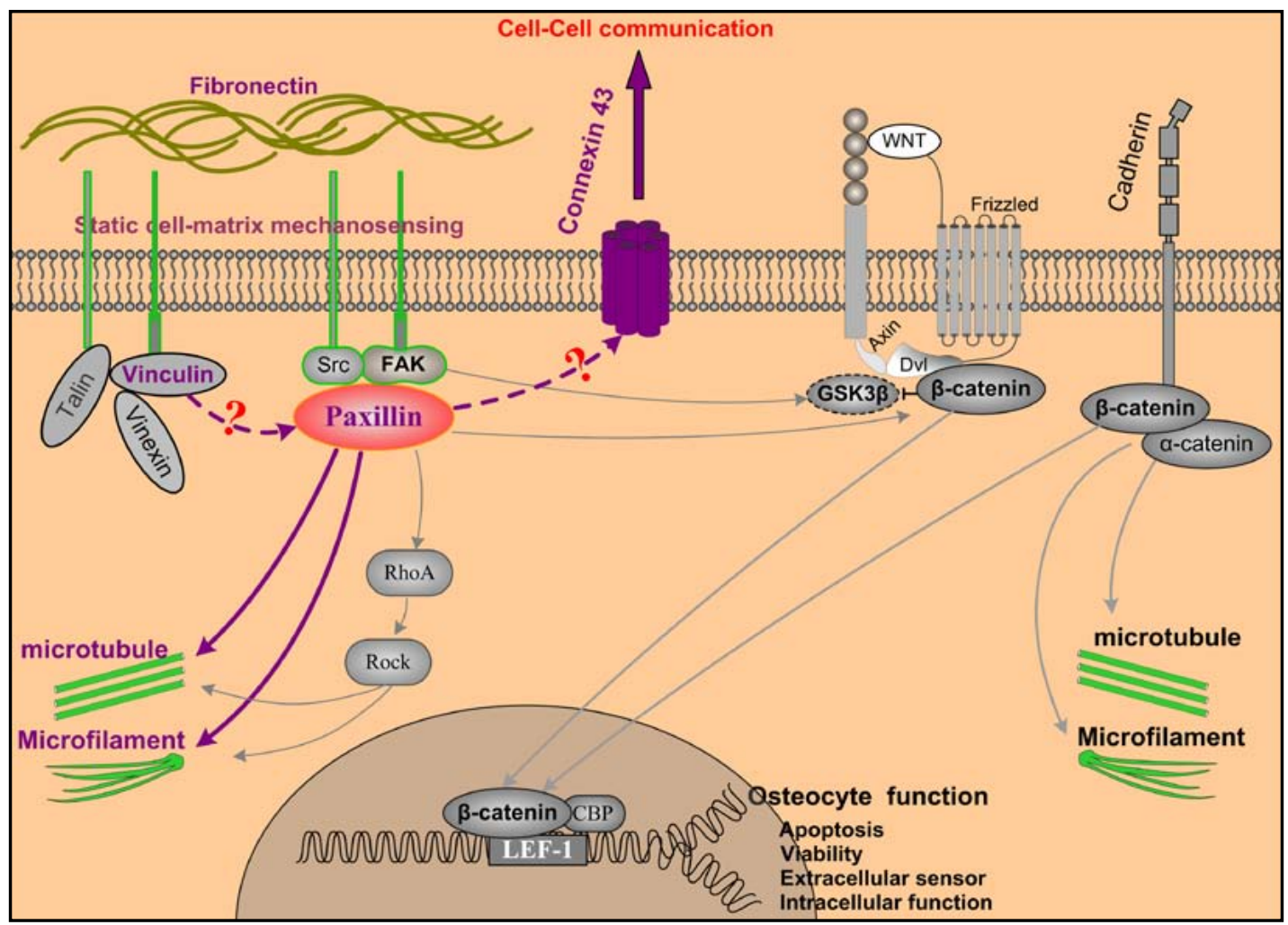

Fig. 6. Schematic diagram explaining how matrix stiffness modulates cytoskeleton rearrangements and the cell function of osteocytes through paxillin. The grey parts are involved in cytoskeleton rearrangements, although not shown in this study, and the green arrows indicate the paxillin-modulated pathway.

cells [33]. In the current study, we favored the concept that the mechanosensing is initiated on the cell body. We provide evidence of changes in vinculin, a membrane-cytoskeletal protein in focal adhesion plaques that is in charge of linking integrin adhesion molecules to the actin cytoskeleton, on the cell body of osteocytes. We further provide proof of an interaction between fibronectin, a linker protein between the ECM and cell membrane, and paxillin, a signaling transduction adaptor protein located on the inside of the cell membrane. These changes in response to substrate stiffness prove that mechanosensing and mechanotransduction occur on the cell body.

Paxillin is a signaling transduction adaptor that activates cytoplasmic signals such as Wnts [34] and can cause changes in downstream targets [35]. Moreover, paxillin can directly alter the cytoskeleton via the reorganization and recombination of microfilaments and microtubules that alter cell responses [36]. In this study, we detected changes in paxillin expression and distribution in response to different substrate stiffnesses and thus inferred the activation of cytoplasmic signaling and the reorganization of the cytoskeleton. Importantly, we further found an interaction between paxillin and connexin 43 proteins (Fig. $5 \mathrm{~A}$ ). The gene and protein expressions of connexin 43 were reduced in accordance with a decrease in the substrate stiffness (Fig. 5B-I). The results indicate that a change in connexin 43 is directly related to a change in paxillin. Because gap junctions are mainly formed by connexin subunits and connexin 43 , encoded by the gap junction alpha 1 (Gja1) gene, is the most abundant connexin in osteocytes, the change in connexin 43 induced by substrate stiffness may represent a change in the gap junction. The results from western blot showed that the total amount of connexin 43 decreased as the substrate stiffness decreased (Fig. 5BD). CLSM images showed that connexin 43 is expressed in the cytoplasm, especially inside the cytoplasm along the cytoskeleton (Fig. 5E). Once a gap junction is formed between two cells, connexin 43 is expressed along the gap junction and fuses together into a completed

\section{KARGER}




\section{Cellular Physiology Cell Physiol Biochem 2018;51:1013-1026

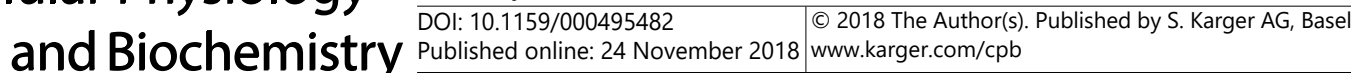 \\ Zhang et al.: Paxillin is Important in Stiffness-Triggered Gap Junction}

linear pattern. Thus, the numbers, lengths, and widths of fused connexin 43 between two cells directly reflect the gap junctions between osteocytes (Fig. 5F, boxed area, and Fig. 5GI). These results indicate that paxillin can regulate gap junction formation and elongation, which suggest its novel and critical role in the communication between osteocytes. Although paxillin-regulated cytoplasmic signals and cytoskeleton alterations can provoke a cascade of events that might involve gap junction modulation, the interaction between paxillin and connexin 43 provides direct evidence of the role of paxillin in gap junction formation and elongation.

One potential limitation of this study is that we only focused on the effect of substrate stiffness. The reality is that osteocytes commonly display both temporal and spatial variations in the level of the mechanical stimuli they exert on their ECM adhesions [37, 38]. The response of osteocytes should be an integrated effect that is not only due to multi-mechanical stimuli, but also multi-chemical factors. The other limitation is that we mimicked variations in ECM stiffness by using PDMS substrates in vitro, but the natural ECM of osteocytes is actually a complex three-dimensional fibrous meshwork with a wide distribution of fibers and gaps that provide complex biochemical and physical cues [39]. This is very different from the uniformly coated surfaces of PDMS substrates, and cell reactions to three-dimensional matrices are different from those to two-dimensional matrices. This factor has to be considered when comparing the stiffened matrix data with the in vivo animal model data or preclinical data.

\section{Acknowledgements}

This work was supported by NSFC grants $(81600840,81771047)$ to Jing Xie. We acknowledge Dr. Chenghui Li in the Analytical \& Testing Center of Sichuan University for her support with the CLSM imaging (A1R MP+, Nikon, Tokyo, Japan).

Demao Zhang and Jing Xie contributed to study design, material PDMS fabrication, cell culture, molecular experiments, and manuscript writing. Demao Zhang, Chenchen Zhou, and Linyi Cai contributed to data collection and analysis. Qingxuan Wang, Wei Du, and Xiaobing Li performed additional experiments and analyses of gene and protein data to supplement the figures. Jing Xie critically reviewed and approved the final manuscript.

\section{Disclosure Statement}

The authors declare no competing financial interests.

\section{References}

-1 Engler AJ, Sen S, Sweeney HL, Discher DE: Matrix elasticity directs stem cell lineage specification. Cell 2006;126:677-689.

-2 Jeong SI, Kwon JH, Lim JI, Cho SW, Jung Y, Sung WJ, Kim SH, Kim YH, Lee YM, Kim BS, Choi CY, Kim SJ: Mechano-active tissue engineering of vascular smooth muscle using pulsatile perfusion bioreactors and elastic plcl scaffolds. Biomaterials 2005; 26:1405-1411.

3 Du J, Chen X, Liang X, Zhang G, Xu J, He L, Zhan Q Feng XQ Chien S, Yang C: Integrin activation and internalization on soft ECM as a mechanism of induction of stem cell differentiation by ECM elasticity. Proc Natl Acad Sci U S A 2011;108:9466-9471.

-4 Chou SY, Cheng CM, Leduc PR: Composite polymer systems with control of local substrate elasticity and their effect on cytoskeletal and morphological characteristics of adherent cells. Biomaterials 2009;30:3136-3142.

-5 Murphy WL, McDevitt TC, Engler AJ: Materials as stem cell regulators. Nat Mater 2014;13:547-557. 


\section{Cellular Physiology Cell Physiol Biochem 2018;51:1013-1026

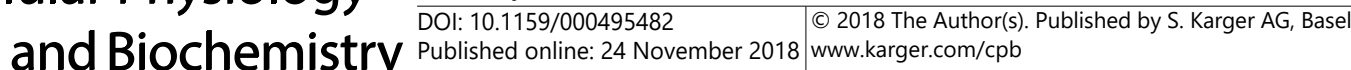

6 Moore KA, Polte T, Huang S, Shi B, Alsberg E, Sunday ME, Ingber DE: Control of basement membrane remodeling and epithelial branching morphogenesis in embryonic lung by Rho and cytoskeletal tension. Dev Dyn 2005;232:268-281.

7 Kumar S, Maxwell IZ, Heisterkamp A, Polte TR, Lele TP, Salanga M, Mazur E, Ingber DE: Viscoelastic retraction of single living stress fibers and its impact on cell shape, cytoskeletalorganization, and extracellular matrix mechanics. Biophys J 2006;90:3762-3773.

-8 Hayes AJ, Benjamin M, Ralphs JR: Role of actin stress fibres in the development of the intervertebral disc: cytoskeletal control of extracellular matrix assembly. Dev Dyn 1999;215:179-189.

-9 Paszek MJ, Zahir N, Johnson KR, Lakins JN, Rozenberg GI, Gefen A, Reinhart-King CA, Margulies SS, Dembo M, Boettiger D, Hammer DA, Weaver VM: Tensional homeostasis and the malignant phenotype. Cancer Cell 2005;8:241-254.

10 Uda Y, Azab E, Sun N, Shi C, Pajevic PD: Osteocyte Mechanobiology. Curr Osteoporos Rep 2017;15:318-325.

11 Vogel V, Sheetz M: Local force and geometry sensing regulate cell functions. Nat Rev Mol Cell Biol 2006;7:265-275.

12 Pal S: Mechanical properties of biological materials. Design of Artifi cial Human Joints \& Organs. 2014;2340.

13 Hemmatian H, Bakker AD, Klein-Nulend J, van Lenthe GH: Aging, Osteocytes, and Mechanotransduction. Curr Osteoporos Rep 2017;15:401-411.

14 You L, Temiyasathit S, Lee P, Kim CH, Tummala P, Yao W, Kingery W, Malone AM, Kwon RY, Jacobs CR: Osteocytes as mechanosensors in the inhibition of bone resorption due to mechanical loading. Bone 2008;42:172-179.

$>15$ Lee K, Jessop H, Suswillo R, Zaman G, Lanyon L: Endocrinology: bone adaptation requires oestrogen receptor-alpha. Nature 2003;424:389.

-16 Terai K, Takano-Yamamoto T, Ohba Y, Hiura K, Sugimoto M, Sato M, Kawahata H, Inaguma N, Kitamura Y, Nomura S: Role of osteopontin in bone remodeling caused by mechanical stress. J Bone Miner Res 1999;14:839-849.

17 Tatsumi S, Ishii K, Amizuka N, Li M, Kobayashi T, Kohno K, Ito M, Takeshita S, Ikeda K: Targeted ablation of osteocytes induces osteoporosis with defective mechanotransduction. Cell Metab 2007;5:464-475.

18 Xie J, Zhang Q Zhu T, Zhang Y, Liu B, Xu J, Zhao H: Substrate stiffness-regulated matrix metalloproteinase output in myocardial cells and cardiacfibroblasts: implications for myocardial fibrosis. Acta Biomater 2014;10:2463-2472.

19 Zhang MG, Cao YP, Li GY, Feng XQ: Spherical indentation method for determining the constitutive parameters of hyperelastic softmaterials. Biomech Model Mechanobiol 2014;13:1-11.

20 Xie J, Zhang D, Lin Y, Yuan Q, Zhou X: Anterior Cruciate Ligament Transection-Induced Cellular and Extracellular Events in Menisci: Implications for Osteoarthritis. Am J Sports Med. 2018;46:1185-1198.

21 Mouw JK, Ou G, Weaver VM: Extracellular matrix assembly: a multiscale deconstruction. Nat Rev Mol Cell Biol 2014;15:771-785.

-22 Wierzbicka-Patynowski I, Schwarzbauer JE: The ins and outs of fibronectin matrix assembly. J Cell Sci 2003;116:3269-3276.

-23 Matsuo K: Cross-talk among bone cells. Curr Opin Nephrol Hypertens 2009;18:292-297.

$\checkmark 24$ Kamioka H, Ishihara Y, Ris H, Murshid SA, Sugawara Y, Takano-Yamamoto T, Lim SS: Primary cultures of chick osteocytes retain functional gap junctions between osteocytes and between osteocytes and osteoblasts. Microsc Microanal 2007;13:108-117.

25 Ishihara Y, Kamioka H, Honjo T, Ueda H, Takano-Yamamoto T, Yamashiro T: Hormonal, pH, and calcium regulation of connexin 43-mediated dye transfer in osteocytes in chick calvaria. J Bone Miner Res 2008;23:350-360.

26 Handorf AM, Zhou Y, Halanski MA, Li WJ: Tissue stiffness dictates development, homeostasis, and disease progression. Organogenesis 2015;11:1-15.

27 Mège RM, Gavard J, Lambert M: Regulation of cell-cell junctions by the cytoskeleton. Curr Opin Cell Biol 2006;18:541-548.

28 Lu P, Takai K, Weaver VM, Werb Z: Extracellular matrix degradation and remodeling in development and disease. Cold Spring Harb Perspect Biol 2011;3: pii: a005058.

-29 Seltmann K, Fritsch AW, Käs JA, Magin TM: Keratins significantly contribute to cell stiffness and impact invasive behavior. Proc Natl Acad Sci U S A 2013;110:18507-18512. 


\section{Cellular Physiology Cell Physiol Biochem 2018;51:1013-1026 and Biochemistry $\begin{gathered}\text { DOl: 10.1159/000495482 } \\ \text { Published }\end{gathered}$ \\ Zhang et al.: Paxillin is Important in Stiffness-Triggered Gap Junction}

30 Bonewald LF: Mechanosensation and Transduction in Osteocytes. Bonekey Osteovision 2006;3:7-15.

-31 Han Y, Cowin SC, Schaffler MB, Weinbaum S: Mechanotransduction and strain amplification in osteocyte cell processes. Proc Natl Acad Sci U S A 2004;101:16689-16694.

-32 Nicolella DP, Feng JQ, Moravits DE, Bonivitch AR, Wang Y, Dusecich V, Yao W, Lane N, Bonewald LF: Effects of nanomechanical bone tissue properties on bone tissue strain: implications for osteocyte mechanotransduction. J Musculoskelet Neuronal Interact 2008;8:330-331.

-33 Malone AM, Anderson CT, Tummala P, Kwon RY, Johnston TR, Stearns T, Jacobs CR: Primary cilia mediate mechanosensing in bone cells by a calcium-independent mechanism. Proc Natl Acad Sci U S A 2007;104:13325-13330.

-34 Matsumoto S, Fumoto K, Okamoto T, Kaibuchi K, Kikuchi A: Binding of APC and dishevelled mediates Wnt5a-regulated focal adhesion dynamics in migrating cells. EMBO J 2010;29:1192-1204.

-35 Turner CE: Paxillin interactions. J Cell Sci 2000;113:4139-4140.

-36 Meehan S, Nain AS: Role of suspended fiber structural stiffness and curvature on single-cell migration, nucleus shape, and focal-adhesion-cluster length. Biophys J 2014;107:2604-2611.

-37 Beningo KA, Dembo M, Kaverina I, Small JV, Wang YL: Nascent focal adhesions are responsible for the generation of strong propulsive forces in migrating fibroblasts. J Cell Biol 2001;153:881-888.

-38 Overby DR, Matthews BD, Alsberg E, Ingber DE: Novel dynamic rheological behavior of individual focal adhesions measured within single cellsusing electromagnetic pulling cytometry. Acta Biomater 2005;1:295-303.

-39 Cukierman E, Pankov R, Stevens DR, Yamada KM: Taking cell-matrix adhesions to the third dimension. Science 2001;294:1708-1712. 